\title{
OBTAINING AND INVESTIGATION OF THE INTEGRAL REPRESENTATION OF SOLUTION AND BOUNDARY INTEGRAL EQUATION FOR THE NON-STATIONARY PROBLEM OF THERMAL CONDUCTIVITY
}

\author{
Grigoriy Zrazhevsky \\ Department of Theoretical Mechanics \\ National Taras Shevchenko University of Kiev \\ 64 Volodymyrs'ka str., Kyiv, Ukraine, 01033 \\ zrazhevsky@aorda.com \\ Vera Zrazhevska \\ Department of Differential Equations \\ National Technical University of Ukraine "Igor Sikorsky Kyiv Polytechnic Institute \\ 37 Peremogi ave., Kyiv, Ukraine, 03056 \\ vera.zrazhevska@gmail.com
}

\begin{abstract}
Technological processes in the energy sector and engineering require the calculation of temperature regime of functioning of different constructions. Mathematical model of thermal loading of constructions is reduced to a non-stationary initial-boundary value problem of thermal conductivity. The article examines the formulation of the non-stationary initial-boundary value problem of thermal conductivity in the form of a boundary integral equation, analyzes the singular equation and builds the fundamental solution. To build the integral representation of the solution the method of weighted residuals is used. The correctness of the obtained integral representation of the solution in Minkowski space is confirmed. Singularity of the fundamental solution is investigated. The boundary integral equation and fundamental solution for axially symmetric domain for internal problem is built. The results of the article can be useful for numerical implementation of boundary element method.

Keywords: initial-boundary problem of thermal conductivity, boundary integral equation, method of weighted residuals, fundamental solution.

\section{Introduction}

Intensive non-stationary thermal load of metal constructions can cause damage and destruction. Processes in energy and engineering provide regular and emergency changes of temperature regimes. Since cylindrical structures, such as pipelines, reactor vessel, constitute a substantial part of the production elements, calculation of critical temperature modes of their operation is important. Theory of temperature stress and engineering methods of their calculation is widely represented in the literature [1-4]. Mathematical model of thermal stress of metal constructions can be reduced to investigation of the non-stationary behavior of thermal fields by solving non-stationary initial-boundary problem of thermal conductivity and calculating of temperature stress by solving stationary boundary problem of elasticity [1].

Analytical solutions of basic model problems for canonical domains are obtained [1-4]. At the same time, calculations and control of real constructions require the development of efficient numerical methods that allow to evaluate the behavior of structures under the influence of intense thermal loads in real time. One of these methods is the boundary element method that is one of numerous implementations of the method of boundary integral equations [5-9].

The method of boundary integral equations is based on the transition from non-stationary initial-boundary problem to the equivalent integral equation. This approach has numerous advantages over classical methods of potential theory because it allows to reduce the dimension of the problem, the solution obtained by this method is semi analytical, and so it can be used for analytical analysis $[5,6,8]$.
\end{abstract}


The method uses the Green function as a fundamental solution. This allows to satisfy one part of the boundary conditions and the condition at infinity (if the infinity is included). The flexibility of the method of boundary integral equations is also evident in the possibility of building special boundary elements that take into account the asymptotic behavior of the solution in a neighborhood of singular points of the problem $[8,9]$. At the same time, the method of boundary integral equation leads to the need to solve singular and hypersingular integral equations, and therefore requires deep analytical study.

The aim of this study is to obtain an integral representation of the solution in the Minkowski space, that allows to get a solution in the form of the boundary integral equation for a boundary value problem. This representation is convenient for the development of numerical methods, based on the decision boundary method of solving the boundary initial value problems of this type [10].

\section{Materials and methods}

\section{1. Problem statement}

Introduce the following notations: $\mathrm{x}=\left(\mathrm{x}_{1}, \mathrm{x}_{2}, \mathrm{x}_{3}\right) \in \mathrm{R}^{3}, \Omega \subset \mathrm{R}^{3}$ is a space domain with the lateral surface $\Gamma=\Gamma_{\mathrm{u}} \cup \Gamma_{\mathrm{q}}, \mathrm{t}$ is time; $\mathrm{u}(\mathrm{x}, \mathrm{t})$ is a temperature at a point $\mathrm{x}$ at a time moment $\mathrm{t}$; $\mathrm{c}(\mathrm{x})$ is the heat capacity, $k(x)$ is the thermal conductivity; $\rho(x)$ is the matter density, $f(x, t)$ is the intensity of heat sources at a point $\mathrm{x}$ at a time moment $\mathrm{t}$.

Consider the problem for the heat equation:

$$
\mathrm{c} \rho \frac{\partial \mathrm{u}}{\partial \mathrm{t}}(\mathrm{x}, \mathrm{t})=\operatorname{div}(\mathrm{k} \operatorname{grad} \mathrm{u}(\mathrm{x}, \mathrm{t}))+\mathrm{f}(\mathrm{x}, \mathrm{t}), \mathrm{x} \in \Omega, \mathrm{t}>\mathrm{t}_{0}
$$

with the boundary conditions:

$$
\left\{\begin{array}{l}
\mathrm{u}(\mathrm{x}, \mathrm{t})=\overline{\mathrm{u}}(\mathrm{x}, \mathrm{t}), \mathrm{x} \in \Gamma_{\mathrm{u}}, \mathrm{t} \geq \mathrm{t}_{0} \\
\mathrm{q}(\mathrm{x}, \mathrm{t})=\overline{\mathrm{q}}(\mathrm{x}, \mathrm{t}), \mathrm{x} \in \Gamma_{\mathrm{q}}, \mathrm{t} \geq \mathrm{t}_{0}
\end{array}\right.
$$

and initial condition:

$$
\mathrm{fu}\left(\mathrm{x}, \mathrm{t}_{0}\right)=\mathrm{u}_{0}(\mathrm{x}), \mathrm{x} \in \bar{\Omega},
$$

where $\bar{\Omega}=\Omega \cup \Gamma, \mathrm{q}(\mathrm{x}, \mathrm{t})=\mathrm{k} \frac{\partial \mathrm{u}}{\partial \overrightarrow{\mathrm{n}}}, \overrightarrow{\mathrm{n}}$ is an external normal to the surface.

Consider the initial boundary problem (1)-(3) as a boundary problem in Minkowski space $(x, t)$, where $x$ is a point of three-dimensional space, $t$ is time. Obviously, we get the boundary problem for cylinder $\left(\mathrm{x} \in \Omega, \mathrm{t}>\mathrm{t}_{0}\right)$ with boundary conditions: (2) on the lateral surface of the cylinder $\left(\mathrm{x} \in \Gamma_{\mathrm{u}} \cup \Gamma_{\mathrm{q}}, \mathrm{t} \geq \mathrm{t}_{0}\right)$ and (3) on the cylinder base.

\section{2. Application of the method of weighted residuals for building the integral repre- sentation of the solution}

We use the method of weighted residuals to build the integral representation of the solution. For this problem the equation of weighted residuals [10] takes the form:

$$
\begin{aligned}
& \int_{\mathrm{t}_{0}}^{\infty} \mathrm{dt} \int_{\Omega}\left[\operatorname{div}(\mathrm{kgrad} u)-\rho \mathrm{c} \frac{\partial \mathrm{u}}{\partial \mathrm{t}}+\mathrm{f}\right] \mathrm{u} * \mathrm{~d} \Omega_{\mathrm{x}}= \\
& =\int_{\mathrm{t}_{\mathrm{o}}}^{\infty} \mathrm{dt} \int_{\Gamma_{\mathrm{q}}}(\mathrm{q}-\overline{\mathrm{q}}) \mathrm{u} * \mathrm{~d} \Gamma_{\mathrm{x}}+\int_{\mathrm{t}_{0}}^{\infty} \mathrm{dt} \int_{\Gamma_{\mathrm{u}}}(\mathrm{u}-\overline{\mathrm{u}}) \mathrm{q} * \mathrm{~d} \Gamma_{\mathrm{x}},
\end{aligned}
$$


where

$$
\mathrm{q}^{*}=\frac{\partial \mathrm{u}^{*}}{\partial \mathrm{n}} \mathrm{k}
$$

In this case, $\mathrm{u}^{*}$ and $\mathrm{q}^{*}$ are the weight functions in $\Omega$ and $\Gamma$ respectively. According to the potential theory $[1,2]$, the solution of the boundary problem can be represented as the integral representation by capacity (volume, simple and double layer). Therefore, $\mathrm{u}^{*}$ and $\mathrm{q}^{*}$ have to depend on continual vector of parameters that belongs to Minkowski space.

Make transformations in the left side of (4): twice integrate by parts by $\mathrm{x}$ and once by $\mathrm{t}$, use Ostrogradsky-Gauss theorem and equality $\operatorname{div}(\vec{a} \cdot b)=\operatorname{div} \vec{a} \cdot b+\vec{a} g r a d b$. If $u$ is the solution of the problem (1)-(3) then integrals on the right side of (4) are equal 0 . So, we get the integral equivalent of our problem:

$$
\begin{gathered}
\int_{\mathrm{t}_{0}}^{\infty} \mathrm{dt} \int\left[\operatorname{div}\left(\mathrm{kgrad} \mathrm{u}^{*}\right)-\rho \mathrm{c} \frac{\partial \mathrm{u}^{*}}{\partial \mathrm{t}}\right] \mathrm{ud} \Omega_{\mathrm{x}}+\int_{\mathrm{t}_{0}}^{\infty} \mathrm{dt} \int\left[\mathrm{u}_{\Gamma}^{*} \mathrm{q}-\mathrm{uq} \mathrm{q}^{*}\right] \mathrm{d} \Gamma_{\mathrm{x}}-\left.\int_{\Omega} \rho \mathrm{cu} \mathrm{u}^{*} \mathrm{~d} \Omega\right|_{\mathrm{t}_{0}} ^{\infty}+ \\
+\int_{\mathrm{t}_{0}}^{\infty} \mathrm{dt} \int_{\Omega} \mathrm{fu} \mathrm{u}^{*} \mathrm{~d} \Omega_{\mathrm{x}}=0 .
\end{gathered}
$$

Further we consider that $\mathrm{c}, \rho, \mathrm{k}=$ const. We take $\mathrm{u}^{*}$ as the fundamental solution of the heat equation:

$$
\mathrm{k} \Delta_{\mathrm{x}} \mathrm{u}^{*}(\xi, \mathrm{x} ; \tau, \mathrm{t})-\rho \mathrm{c} \frac{\partial \mathrm{u}^{*}}{\partial \mathrm{t}}=\delta(\xi, \mathrm{x}) \delta(\tau, \mathrm{t})
$$

where $\delta(\cdot)$ is the Dirac delta - function. Physical solution of (6) that satisfies the principle of causality and is limited at infinity for four - dimensional Minkowski space has the form [10]:

$$
u^{*}=-\frac{(\rho c)^{1 / 2}}{(4 \pi k(\tau-t))^{3 / 2}} \exp \left(-\frac{|\xi-x|^{2} \rho c}{4 k(\tau-t)}\right) H(\tau-t)
$$

where $\mathrm{H}$ is the Heaviside function. Then (5) can be written as:

$$
\begin{gathered}
\chi_{\left(\Omega, t>t_{0}\right)}(\xi, \tau) \cdot u(\xi, \tau)+\int_{t_{0}}^{\tau} \mathrm{dt} \int_{\Gamma}(\mathrm{u} *(\xi-\mathrm{x}, \tau-\mathrm{t}) \mathrm{q}(\mathrm{x}, \mathrm{t})-\mathrm{u}(\mathrm{x}, \mathrm{t}) \mathrm{q} *(\mathrm{x}-\xi, \tau-\mathrm{t})) \mathrm{d} \Gamma_{\mathrm{x}}= \\
=\int_{\Omega} \rho \mathrm{cu} \mathrm{u}_{0}(\mathrm{x}) \mathrm{u} *\left(\xi-\mathrm{x}, \tau-\mathrm{t}_{0}\right) \mathrm{d} \Omega_{\mathrm{x}}-\int_{\mathrm{t}_{0}}^{\tau} \mathrm{dt} \int_{\Omega} \mathrm{f}(\mathrm{x}, \mathrm{t}) \mathrm{u} *(\xi-\mathrm{x}, \tau-\mathrm{t}) \mathrm{d} \Omega_{\mathrm{x}},
\end{gathered}
$$

where

$$
\chi_{\left(\Omega, t>t_{0}\right)}(\xi, t)=\left\{\begin{array}{l}
1,(\xi, t) \in\left(\Omega, t>t_{0}\right) \\
0,(\xi, t) \notin\left(\Omega, t>t_{0}\right)
\end{array}\right.
$$

is the area function (indicator).

It is clear that (8) is the quasi equation. If we know values of functions $\mathrm{u}(\mathrm{x}, \mathrm{t})$ and $\mathrm{q}(\mathrm{x}, \mathrm{t})$ on $\Gamma$, (8) allows to determine $\mathrm{u}(\mathrm{x}, \mathrm{t})$ at any point inside the area $\left(\mathrm{x} \in \Omega, \mathrm{t}>\mathrm{t}_{0}\right)$. So, the problem is to determine $\mathrm{u}(\mathrm{x}, \mathrm{t})$ on $\Gamma_{\mathrm{q}}$ and $\mathrm{q}(\mathrm{x}, \mathrm{t})$ on $\Gamma_{\mathrm{u}}$.

To prove the correctness of constructing of the integral representation of the solution in the form (8), we show that: 


$$
\lim _{\tau \rightarrow \infty} \int_{0}^{\tau} \mathrm{u} *(\xi-\mathrm{x}, \tau-\mathrm{t}) \mathrm{dt}=\hat{\mathrm{u}}(\xi, \mathrm{x})
$$

where $\hat{\mathrm{u}}(\xi, \mathrm{x})$ is up to a constant a fundamental solution of the Laplace operator

$$
\left(\Delta_{\mathrm{x}}=\frac{\partial^{2} \mathrm{u}}{\partial \mathrm{x}_{1}^{2}}+\frac{\partial^{2} \mathrm{u}}{\partial \mathrm{x}_{2}^{2}}+\frac{\partial^{2} \mathrm{u}}{\partial \mathrm{x}_{3}^{2}}\right)
$$

Really

$$
\begin{aligned}
\int_{0}^{\tau} \mathrm{u} *(\xi-\mathrm{x}, \tau-\mathrm{t}) \mathrm{dt} & =-\frac{(\rho \mathrm{c})^{1 / 2}}{(4 \pi \mathrm{k})^{3 / 2}} \int_{0}^{\tau} \frac{1}{(\tau-\mathrm{t})^{3 / 2}} \exp \left(-\frac{|\xi-\mathrm{x}|^{2} \rho \mathrm{c}}{4 \mathrm{k}(\tau-\mathrm{t})}\right) \mathrm{dt}=\left[\mathrm{s}=\frac{|\xi-\mathrm{x}|^{2} \rho \mathrm{c}}{4 \mathrm{k}(\tau-\mathrm{t})}\right]= \\
& =-\frac{1}{4 \mathrm{k} \pi^{3 / 2}|\xi-\mathrm{x}|} \int_{\mathrm{a}}^{\infty} \mathrm{s}^{-1 / 2} \mathrm{e}^{-\mathrm{s}} \mathrm{ds}=\frac{\Gamma(1 / 2, \mathrm{a})}{4 \mathrm{k} \pi^{3 / 2}|\xi-\mathrm{x}|},
\end{aligned}
$$

where $\mathrm{a}=\frac{|\xi-\mathrm{x}|^{2} \rho \mathrm{c}}{4 \mathrm{k} \tau}, \Gamma(\cdot, \cdot)$ is an incomplete gamma function.

If $\tau \rightarrow \infty$, then $\mathrm{a} \rightarrow 0$ and $\Gamma(1 / 2, \mathrm{a}) \rightarrow \Gamma(1 / 2)=\pi^{1 / 2}$. So, $\int_{0}^{\tau} \mathrm{u}^{*} \mathrm{dt} \rightarrow \frac{1}{4 \mathrm{k} \pi|\xi-\mathrm{x}|}$, and the function $\hat{\mathrm{u}}(\xi, \mathrm{x})=\frac{1}{4 \mathrm{k} \pi|\xi-\mathrm{x}|}$ is the solution of the equation $\mathrm{k} \Delta_{\mathrm{x}} \hat{\mathrm{u}}(\xi-\mathrm{x})=\delta(\xi, \mathrm{x})$.

\section{3. Construction of a boundary integral equation of the problem}

To get a boundary integral equation we take the limit in (8) as $\xi \rightarrow \bar{\xi} \in \Gamma$. Since the integral

$$
\int_{t_{0}}^{\tau} \mathrm{dt} \int_{\Gamma} \mathrm{u}^{*}(\bar{\xi}-\mathrm{x}, \tau-\mathrm{t}) \mathrm{q}(\mathrm{x}, \mathrm{t}) \mathrm{d} \Gamma_{\mathrm{x}}
$$

is not special, we consider only the singular integral

$$
\int_{t_{0}}^{\tau} \mathrm{dt} \int_{\Gamma} \mathrm{u}(\mathrm{x}, \mathrm{t}) \mathrm{q}^{*}(\mathrm{x}-\bar{\xi}, \tau-\mathrm{t}) \mathrm{d} \Gamma_{\mathrm{x}}
$$

We will determine this integral in the sense of Cauchy principal value (v.p.) in four- dimensional space $(\mathrm{x}, \mathrm{t})$.

Complement $\Gamma$ with the spherical surface $\Gamma_{\varepsilon}$ with center in $\bar{\xi}$ and radius $\varepsilon<<1$. Throwing the part of $\Gamma$ which is inside $\Gamma_{\varepsilon}$, we get a new border $\bar{\Gamma}_{\varepsilon}$, so that $\bar{\Gamma}_{\varepsilon} \rightarrow \Gamma$ when $\varepsilon \rightarrow 0$. Then

$$
\begin{gathered}
\int_{\mathrm{t}_{0}}^{\tau} \mathrm{dt} \int_{\Gamma} \mathrm{u}(\mathrm{x}, \mathrm{t}) \mathrm{q}^{*}(\mathrm{x}-\bar{\xi}, \tau-\mathrm{t}) \mathrm{d} \Gamma_{\mathrm{x}}=\lim _{\substack{\varepsilon \rightarrow 0 \\
\chi \rightarrow 0}}\left(\int_{\mathrm{t}_{0}}^{\tau-\chi} \mathrm{dt} \int_{\bar{\Gamma}_{\varepsilon} \Gamma_{\varepsilon}} \mathrm{u}(\mathrm{x}, \mathrm{t}) \mathrm{q}^{*}(\mathrm{x}-\bar{\xi}, \tau-\mathrm{t}) \mathrm{d} \Gamma_{\mathrm{x}}+\right. \\
\left.+\int_{\tau-\chi}^{\tau} \mathrm{dt} \int_{\Gamma_{\varepsilon}} \mathrm{u}(\mathrm{x}, \mathrm{t}) \mathrm{q}^{*}(\mathrm{x}-\bar{\xi}, \tau-\mathrm{t}) \mathrm{d} \Gamma_{\mathrm{x}}\right)
\end{gathered}
$$

where $\lim _{\substack{\varepsilon \rightarrow 0 \\ \chi \rightarrow 0}} \int_{\mathrm{t}_{0}}^{\tau-\chi} \mathrm{dt} \int_{\bar{\Gamma}_{\varepsilon} \backslash \Gamma_{\varepsilon}} \mathrm{u}(\mathrm{x}, \mathrm{t}) \mathrm{q}^{*}(\mathrm{x}-\bar{\xi}, \tau-\mathrm{t}) \mathrm{d} \Gamma_{\mathrm{x}}=\mathrm{v} \cdot \mathrm{p} \cdot \int_{\mathrm{t}_{0}}^{\tau} \mathrm{dt} \int_{\Gamma} \mathrm{u}(\mathrm{x}, \mathrm{t}) \mathrm{q}^{*}(\mathrm{x}-\bar{\xi}, \tau-\mathrm{t}) \mathrm{d} \Gamma_{\mathrm{x}} \quad$ (v.p. hereinafter we will miss). For $\chi<<1, \varepsilon<<1$, to integral $I_{\varepsilon, \chi}(\bar{\xi}, \tau)=\int_{\tau-\chi}^{\tau} \operatorname{dt} \int_{\Gamma_{\varepsilon}} u(x, t) q^{*}(x-\bar{\xi}, \tau-t) d \Gamma_{x}$ we use the average theorem $(u(\bar{\xi}, \tau)$ is limited $)$ : 


$$
\mathrm{I}_{\varepsilon, \chi}(\bar{\xi}, \tau)=\mathrm{u}(\tilde{\xi}, \tilde{\tau}) \int_{\tau-\chi}^{\tau} \mathrm{dt} \int_{\Gamma_{\varepsilon}} \mathrm{q}^{*}(\mathrm{x}-\bar{\xi}, \tau-\mathrm{t}) \mathrm{d} \Gamma_{\mathrm{x}}
$$

where $\bar{\xi} \in \Gamma_{\varepsilon}, \tilde{\tau} \in[\tau-\chi, \tau]$.

Using (7) and taking into account that in the spherical coordinate system centered at $\bar{\xi}$ at each point on the surface $\left.\Gamma_{\varepsilon} \vec{n}\right|_{\Gamma_{\varepsilon}}=\vec{r}$, we have:

$$
\mathrm{I}_{\varepsilon, \chi}(\bar{\xi}, \tau)=\mathrm{u}(\tilde{\xi}, \tilde{\tau})(-1) \frac{(\rho \mathrm{c})^{1 / 2}}{(4 \pi \mathrm{k})^{3 / 2}} \int_{\tau-\chi}^{\tau} \frac{(-1) \rho \mathrm{c} 2}{4 \mathrm{k}(\tau-\mathrm{t})} \frac{\varepsilon \mathrm{k}}{(\tau-\mathrm{t})^{3 / 2}} \exp \left(-\frac{\varepsilon^{2} \rho \mathrm{c}}{4 \mathrm{k}(\tau-\mathrm{t})}\right) \varepsilon^{2} \mathrm{Sdt}
$$

where $\mathrm{S}$ is the square of segment of unit radius sphere outside of $\Omega$ that is cutting off by $\Gamma$. If $\bar{\xi}$ is a regular point, then $S=2 \pi$ and for variable $v=\frac{\varepsilon^{2} \rho c}{4 k(\tau-t)}(10)$ can be written as:

$$
\mathrm{I}_{\varepsilon, \chi}(\bar{\xi}, \tau)=\mathrm{u}(\tilde{\xi}, \tilde{\tau}) \frac{(\rho \mathrm{c})^{3 / 2} 2 \varepsilon}{(4 \pi \mathrm{k})^{3 / 2} 4 \mathrm{k}} \varepsilon^{2} \operatorname{Sk} \int_{\mathrm{a}}^{\infty} \mathrm{v}^{5 / 2} \frac{\varepsilon^{2} \rho \mathrm{c}}{\mathrm{kv}^{2}} \mathrm{e}^{-\mathrm{v}} \mathrm{dv} \text {, or } \mathrm{I}_{\varepsilon, \chi}(\bar{\xi}, \tau)=\frac{1}{4 \pi^{3 / 2}} \mathrm{u}(\tilde{\xi}, \tilde{\tau}) \operatorname{S} \Gamma(1 / 2, \mathrm{a})
$$

where $\mathrm{a}=\frac{\varepsilon^{2} \rho \mathrm{c}}{4 \mathrm{k} \chi}$.

If $\varepsilon \rightarrow 0$ and $\chi \rightarrow 0$, then $\left(\varepsilon^{2} / \chi\right) \rightarrow 0, \mathrm{a} \rightarrow 0$. In this case

$$
\mathrm{I}_{\varepsilon, \chi}(\bar{\xi}, \tau) \underset{\substack{\varepsilon \rightarrow 0 \\ \chi \rightarrow 0}}{\longrightarrow} \frac{\mathrm{S}}{4 \pi} \mathrm{u}(\bar{\xi}, \tau)
$$

Thus, the dimensionless radius of the sphere in four-dimensional space is $\varepsilon^{2} / \chi$. So, the boundary integral equation of the problem takes form:

$$
\begin{gathered}
\mathrm{C}(\bar{\xi}) \mathrm{u}(\bar{\varepsilon}, \tau)+\int_{\mathrm{t}_{0}}^{\tau} \mathrm{dt} \int_{\Gamma}(\mathrm{u} *(\bar{\xi}-\mathrm{x}, \tau-\mathrm{t}) \mathrm{q}(\mathrm{x}, \mathrm{t})-\mathrm{u}(\mathrm{x}, \mathrm{t}) \mathrm{q} *(\bar{\xi}-\mathrm{x}, \tau-\mathrm{t})) \mathrm{d} \Gamma_{\mathrm{x}}= \\
=\int_{\Omega} \rho \mathrm{c} \mathrm{u}_{0}(\mathrm{x}) \mathrm{u} *\left(\bar{\xi}-\mathrm{x}, \tau-\mathrm{t}_{0}\right) \mathrm{d} \Omega_{\mathrm{x}}-\int_{\mathrm{t}_{0}}^{\tau} \mathrm{dt} \int_{\Omega} \mathrm{f}(\mathrm{x}, \mathrm{t}) \mathrm{u} *(\bar{\xi}-\mathrm{x}, \tau-\mathrm{t}) \mathrm{d} \Omega_{\mathrm{x}},
\end{gathered}
$$

where $C(\bar{\xi})=\frac{1}{4 \pi}\left(4 \pi-S\right.$ ) (if $\bar{\xi}$ is regular then $C(\bar{\xi})=\frac{1}{2}$ ) and the second integral in the left side is taken in the sense of principal value.

Thus, the original problem (1)-(3) is reduced to solving of the singular integral equation (11), that is, to finding $\mathrm{u}(\mathrm{x}, \mathrm{t})$ for $\mathrm{x} \in \Gamma_{\mathrm{q}}$ and $\mathrm{q}(\mathrm{x}, \mathrm{t})$ for $\mathrm{x} \in \Gamma_{\mathrm{u}}$. Then the integral representation of the solution (8) can be used to find $u(\xi, \tau)$ for $(\xi, \tau) \in\left(\Gamma, \tau>t_{0}\right)$. It should be emphasized that (11) is an equation in three-dimensional space $\left(\bar{\xi} \in \Gamma, \tau>t_{0}\right)$, and not in four-dimensional space $\left(\xi \in \mathrm{R}, \tau>\mathrm{t}_{0}\right)$ as the original problem. So, the dimension of the problem is reduced. So, the transition to the boundary integral equation allows to reduce the dimension of the problem.

\section{Results}

The paper considers the initial-boundary value problem for the heat equation in the four-dimensional Minkowski space. The problem is reformulated as a singular boundary integral equation on the four-dimensional cylindrical surface. This approach is useful in the development of numerical methods, because it allows to standardize the dimensions and solve the boundary problem for a fixed spatial region and for a region with a boundary variable in time. However, this approach required the additional study of the behavior of the fundamental solution, and the interpretation of singular integrals in the boundary integral equation. The method of weighted residuals was used to get the integral representation of the solution and the boundary integral. 
The results of the study do not contrary to the classical results of the theory of the initialboundary value problems for parabolic equations, they only give them a new interpretation.

The obtained results can be used in the development of standardized boundary numerical methods.

\section{Conclusions}

The work is devoted to investigation of some aspects of the formulation of the non-stationary initial-boundary value problem of thermal conductivity in the form of a boundary integral equation. In particular, in the work:

- the properties of the physical fundamental solution are defined;

- the integral representation of the solution is constructed and investigated;

- the interpretation of the Cauchy principal value of integral singularity if the point of source approaches the boundary of the four-dimensional domain is conducted, the conditions on the parameters of the passage to the limit for correct interpretation of the singular integral in four-dimensional space are formulated;

- initial-boundary problem is reformulated as a boundary integral equation.

This article proposes a new interpretation of well-known classical results of the theory of partial differential equations. The strict mathematical description of the interpretation of the dynamic initial boundary value problem in the form of a boundary value problem in the Minkowski space is the theoretical value of the research. The obtained results can be extended in two main ways. Firstly, the approach can be applied to other non-stationary problems, for example, in the theory of linear dynamic elasticity. Secondly, such approach can simplify the formalization and solving of the initial-boundary value problems in domains with boundary varying in time, such as non-stationary Stefan problem, free boundary problems, and so on. The method of boundary solutions that is universal for boundary value problems is the practical value of the results. The problem is reduced to finding the unknown functions on the boundary in the Minkowski space, so the actual dimension of the problem is reduced by one that is very significant in building efficient numerical methods, for example, the boundary element method.

\section{References}

[1] Kartashov, E., Kudinov, V. (2012). Analytical theory of heat conduction and thermoelasticity. Librocom, 656 .

[2] Lurie, A. I. (2010). Theory of Elasticity. Springer Science \& Business Media, 1050. doi: 10.1007/978$3-540-26455-2$

[3] Aldo, M. (2010). Theory of Elasticity, 1st Edition. Springer Science \& Business Media, 716. doi: 10.1007/978-3-642-11392-5

[4] Zarubin, V., Kyvurkin, G. (2002). Mathematical models of thermal mechanics. Moscow: Fizmatlit, 168 .

[5] Reddy, J. N. (2006). An Introduction to the Finite Element Method, 3rd Edition. McGraw Hill, 672.

[6] Zienkiewicz, O. C., Taylor, R. L., Zhu, J. Z. (2013). The Finite Element Method: Its Basis and Fundamentals, 7th Edition Butterworth-Heinemann, 712. doi: 10.1016/c2009-0-24909-9

[7] Chaskalovic, J. (2008). Finite Elements Methods for Engineering Sciences, Springer Verlag, 267.

[8] Wrobel, L. C., Aliabadi, M. H. (2002). The Boundary Element Method, New York: John Wiley \& Sons, 1066.

[9] Constanda, C., Doty, D., Hamill, W. (2016). Boundary Integral Equation Methods and Numerical Solutions. Springer, 232. doi: 10.1007/978-3-319-26309-0

[10] Schanz, M., Steinbach, O. (2007). Boundary Element Analysis. Lecture Notes in Applied and Computational Mechanics, 352. doi: 10.1007/978-3-540-47533-0 